\title{
microRNA-125b promotes leukemia cell resistance to daunorubicin by inhibiting apoptosis
}

\author{
LILI ZHOU* ${ }^{*}$ HAITAO BAI ${ }^{*}$, CHUN WANG, DAOLIN WEI, YOUWEN QIN and XIAOWEI XU \\ Department of Hematology, Shanghai Jiaotong University, \\ Affiliated Shanghai First People's Hospital, Shanghai 200080, P.R. China
}

Received August 8, 2013; Accepted February 17, 2014

DOI: $10.3892 / \mathrm{mmr} .2014 .2011$

\begin{abstract}
RNA-125b (miR-125b) is overexpressed in several types of cancer and contributes to tumor resistance to chemotherapy. The present study investigated the effect of miR-125b on the resistance of leukemia cell lines to the chemotherapeutic agent daunorubicin (DNR). miR-125b expression was found to be upregulated in patients who had failed therapy compared with those who demonstrated event-free survival. The overexpression of miR-125b was observed to induce DNR resistance in K562, THP-1 and Jurkat cells by reducing apoptosis, whereas the suppression of miR-125b was found to enhance DNR cytotoxicity in REH cells. Furthermore, miR-125b was observed to mediate DNR resistance in leukemia cell lines through decreasing expression of $\mathrm{G}$ protein-coupled receptor kinase 2 and p53-upregulated modulator of apoptosis, which were shown to be direct targets of miR-125b using a dual-luciferase reporter. The present study provides a novel mechanism for understanding leukemia drug resistance and provides a novel method for calculating patient prognosis.
\end{abstract}

\section{Introduction}

Acute leukemia is a hematological malignant tumor characterized by the rapid accumulation of myeloid blasts in the bone marrow and peripheral blood. Acute leukemia is rapidly fatal without treatment and has a poor prognosis. Over recent decades, the treatment of acute leukemia has developed, with advances in anthracycline-based chemotherapy and stem-cell transplantation $(1,2)$ improving the outlook for patients with acute leukemia. However, although the prognosis of acute

Correspondence to: Professor Chun Wang, Department of Hematology, Shanghai Jiaotong University, Affiliated Shanghai First People's Hospital, 100 Haining Road, Shanghai 200080, P.R. China E-mail: wangchunmd@163.com

*Contributed equally

Key words: microRNA-125b, GRK2, PUMA, daunorubicin, acute leukemia leukemia has improved, the five- and 10-year relative survival estimates for patients with leukemia are only 21.4 and $18.7 \%$, respectively (3). Drug resistance is the primary cause of disease relapse or lack of remission in patients with acute leukemia. Therefore, studies into the mechanisms associated with chemotherapy resistance are required.

microRNAs (miRNAs), a novel class of small noncoding RNAs, ranging between 19 and 25 nucleotides in size, regulate specific target genes through translational repression or direct mRNA degradation. miRNAs thereby regulate numerous cellular functions, including cell proliferation, differentiation and apoptosis (4-6). A previous study showed that the deregulated expression of specific miRNAs that modulate the expression of oncogenes and tumor suppressors is associated with the development of malignancies, and that specific miRNA expression signatures can be used to effectively classify human tumors (7). miR-125b is the ortholog of lin-4 in Caenorhabditis elegans and is highly conserved in numerous species, from nematodes to humans. In humans, there are two homologs: hsa-miR-125b-1 and hsa-miR-125b-2, transcribed from two loci on chromosomes 11q23 and 21q21, respectively. miR-125b has been found to be an oncomiR in hepatocellular carcinoma (8), breast cancer (9), lung cancer (10), melanoma (11) and gastrointestinal cancer (12). Furthermore, miR-125 is involved in myelodysplastic syndrome, acute myeloid leukemia (AML), B-cell acute lymphoid leukemia, megakaryoblastic leukemia and chronic lymphocytic leukemia (CLL) (13-17). In addition, miR-125 contributes to leukemogenesis (18) and increases drug resistance in pediatric acute promyelocytic leukemia (18) and acute lymphoblastic leukemia (ALL) cells treated with vincristine and daunorubicin (DNR) (19).

The present study aimed to investigate the value of miR-125b in determining acute leukemia prognosis and the role of miR-125b in inducing DNR resistance in leukemia cell lines through the inhibition of apoptosis.

\section{Materials and methods}

Patients and sample collection. A total of 46 patients with acute leukemia from Shanghai Jiaotong University Affiliated Shanghai First People's Hospital (Shanghai, China) were enrolled in this study between May 2011 and November 2012. All of the patients were primary or no-remission patients. Patient exclusion criteria were concomitant or previous cancer and acute promyelocytic 
leukemia. The characteristics of the patients included in this study are listed in Table I. Bone marrow was collected from patients by bone marrow puncture at diagnosis or at follow-up subsequent to therapy. All patients were followed for at least four months or until recurrence. Event-free survival (EFS) and overall survival (OS) were the end-points used for the analysis of the treatment results. EFS (20) was measured from the date of entry into the present study to the date of induction failure, relapse from complete remission (CR), CR with incomplete hematologic recovery or death from any cause. OS was calculated from the date of entry into the study to the date of death from any cause. Data were censored if patient had no event at the last follow-up. Healthy samples were collected from three healthy adults. Written informed consent was obtained from the patients for the biological studies. This study was approved by the Ethics Committee of Shanghai Jiaotong University Affiliated Shanghai First People's Hospital.

RNA extraction and quantitative polymerase chain reaction ( $q P C R)$. To evaluate miR-125b expression in bone marrow mononuclear cells, qPCR for miRNA was performed. Mononuclear cells were separated using a Ficoll-Hypaque centrifugation gradient from $2 \mathrm{ml}$ bone marrow samples. Total RNA was isolated with TRIzol ${ }^{\circledR}$ reagent (Invitrogen Life Technologies, Carlsbad, CA, USA) according to the manufacturer's instructions. To detect miR-125b expression, 10 ng total RNA was reverse transcribed with miRNA-specific primers using a TaqMan ${ }^{\circledR}$ MicroRNA Reverse Transcription kit (Applied Biosystems, Foster City, CA, USA). qPCR was then performed using TaqMan ${ }^{\circledR}$ MicroRNA Assays (Applied Biosystems) according to the manufacturer's instructions, using an StepOnePlus ${ }^{\mathrm{TM}}$ Real-Time PCR System (Applied Biosystems). The $20-\mu 1$ PCR reaction contained the following: $1.3 \mu \mathrm{l}$ reverse transcription (RT) product, $10 \mu 1 \mathrm{TaqMan}^{\circledR}$ Universal PCR Master Mix and $1 \mu \mathrm{l}$ primer and probe mix from the TaqMan miRNA assays. The reactions were incubated in optical plates at $95^{\circ} \mathrm{C}$ for $10 \mathrm{~min}$, followed by 40 cycles of $95^{\circ} \mathrm{C}$ for $15 \mathrm{sec}$ and $60^{\circ} \mathrm{C}$ for $10 \mathrm{~min}$. U6 small nuclear RNA was used as a control. miR-125b expression relative to that in a healthy sample was calculated using the $2^{-\Delta \Delta C T}$ method (21). All of the qPCR assays were performed in triplicate.

Cell culture. The K562, THP-1, Jurkat and REH cell lines (The Cell Bank of Type Culture Collection of Chinese Academy of Sciences, Shanghai, China) were cultured in RPMI-1640 medium (HyClone, Logan, UT, USA) supplemented with $10 \%$ fetal bovine serum (FBS; Gibco-BRL, Carlsbad, CA, USA), $100 \mathrm{U} / \mathrm{ml}$ penicillin and $100 \mu \mathrm{g} / \mathrm{ml}$ streptomycin. HEK293T cells (The Cell Bank of Type Culture Collection of Chinese Academy of Sciences, Shanghai, China) were grown in Dulbecco's Modified Eagle Medium (HyClone) containing $10 \% \mathrm{FBS}, 100 \mathrm{~g} / \mathrm{ml}$ L-glutamine, $100 \mathrm{U} / \mathrm{ml}$ penicillin and $100 \mu \mathrm{g} / \mathrm{ml}$ streptomycin.

Overexpression and knockdown of miR-125b. In order to overexpress miR-125b in K562, THP-1 and Jurkat cells, $10 \mu \mathrm{g}$ expression vector or empty vector with $10 \mu \mathrm{g}$ packing plasmid (gag/pol and vesicular stomatitis virus G) was incubated with Fugene $6{ }^{\circledR}$ (Roche, Mannheim, Germany) for $15 \mathrm{~min}$ at room temperature and subsequently added to the HEK293T
Table I. Characteristics of patients with acute leukemia.

\begin{tabular}{lr}
\hline Characteristics & Value \\
\hline Age in years at diagnosis, median (range) & $44(15-67)$ \\
Gender, $\mathrm{n}(\%)$ & \\
Male & $26(56.52)$ \\
Female & $20(43.48)$ \\
WBC count, $(\%)$ & \\
$<4 \times 10^{9} / 1$ & $13(28.26)$ \\
$\geq 4 \times 10^{9} / 1,<10 \times 10^{9} / 1$ & $6(12.77)$ \\
$\geq 10 x 10^{9} / 1,<50 \times 10^{9} / 1$ & $13(28.26)$ \\
$\geq 50 x 10^{9} / 1$ & $14(30.43)$ \\
FAB + immunophenotyping, $\mathrm{n}(\%)$ & \\
ALL & $11(23.91)$ \\
AML & \\
M2 & $8(17.39)$ \\
M4 & $8(17.39)$ \\
M5 & $12(26.09)$ \\
Others & $3(6.50)$ \\
Mixed lineage acute leukemia, $\mathrm{n}(\%)$ & $4(8.70)$ \\
Primary, $\mathrm{n}(\%)$ & $31(67.39)$ \\
Recurrence/no remission, $\mathrm{n}(\%)$ & $15(32.61)$ \\
\hline
\end{tabular}

ALL, acute lymphoma leukemia; AML, acute myelogenous leukemia; WBC, white blood cell; FAB, French-American-British classification.

cells. The viral supernatant was harvested after $48 \mathrm{~h}$, and the cells were treated by a spin infection with retroviral supernatant ( $1 \mathrm{ml}$ supernatant per $1 \times 10^{6}$ cells plus polybrene) in six-well plates that were pre-coated with retronectin (Takara Bio, Inc., Shiga, Japan). The percentage of green fluorescent protein-positive cells was 70-90\%.

In accordance with a previous study (22), miR-125 'sponge' sequences (8-9 tandem repeats, each complementary to miR-125) were designed. An imperfect base-pairing between the miRNA and the miR-125 'sponge' was designed to impair endonucleolytic cleavage by Argonaute-2. The miR-125 'sponge' sequence was 5'-TCACAGGTTACTCAGGGA-3' and was cloned into the pRetroQ-mCherry-C1 retroviral vector (Clontech Laboratories, Inc., Palo Alto, CA, USA). The miR-125 'sponge' vector was then infected into REH cells using a retrovirus, in order to generate miR-125b-knockdown REH cells. Subsequent to virus infection, REH cells were screened using puromycin (Sigma-Aldrich, St. Louis, MO, USA) at an final concentration of $2 \mu \mathrm{g} / \mathrm{ml}$. The percentage of red fluorescent protein-positive cells was $\sim 100 \%$.

Luciferase assay. G protein-coupled receptor kinase (GRK) 2 and p53-upregulated modulator of apoptosis (PUMA) 3' untranslated region (3'UTR) luciferase reporters were individually generated by inserting full-length human GRK2 and PUMA 3'UTR into the XhoI and NotI sites in the psiCHECK-2 vector (Promega, Madison, WI, USA) 
downstream from the Renilla luciferase coding sequence. The GRK2 3'UTR was PCR amplified from human genomic DNA (Roche) using the following primer sequences: Forward, 5'-TCGCTCGAGCCC GCCCACCCGCCTTTTA-3' and reverse, 5'-TCGGCGGCC GCAATCAGGCACCATTTT-3'. The PUMA 3'UTR was PCR amplified using the following primer sequences: Forward, 5'-TCGCTCGAGGACTT TCTCTGCACCAT-3' and reverse, 5'-TAAGCGGCC GCGGCAAGCAGAAAGAGT-3'. The sequences and cloning direction of the PCR products were validated by DNA sequencing. The HEK293T cells were plated in 96-well plates at 5,000 cells/well the day prior to transfection. Transfection was performed in triplicate with Fugene 6 (Roche) and $150 \mathrm{ng}$ plasmid mixture (135 ng miR-125b expression vector and $15 \mathrm{ng}$ reporter vector). Luciferase assays for firefly and Renilla luciferase were performed $48 \mathrm{~h}$ after transfection with a Dual-Glo ${ }^{\circledR}$ Luciferase Assay kit (Promega). Luminescence was quantified using a NOVOstar machine (BMG Labtech, Ortenberg, Germany). The Renilla luciferase readings were normalized to the firefly luciferase activity in the corresponding well.

Cell viability assay. Cells were seeded into 96-well plates in RPMI-1640 medium containing 10\% FBS and were treated with serial dilutions of DNR (Pfizer, Groton, CT, USA). Cell viability was determined $48 \mathrm{~h}$ after DNR treatment using a luminescent cell viability assay (Promega). Luminescence was quantified using a NOVOstar machine (BMG Labtech).

Small interfering (si)RNA transfection. K562 cells were transfected with GRK2 or PUMA siRNA (Santa Cruz Biotechnology, Inc., Santa Cruz, CA, USA) using Fugene 6 (Roche) according to the manufacturer's instructions. The transfection efficiency was $\sim 80 \%$ and was calculated as the percentage of fluorescein-labeled cells using fluorescence microscopy. Forty-eight hours after transfection, the cells were harvested for further analysis.

Western blot analysis. Cells were washed twice with phosphate-buffered saline and then lysed in lysis buffer (Beyotime Institute of Biotechnology, Shanghai, China) containing PhosSTOP (Roche). Cell lysates ( $40 \mu \mathrm{g}$ protein) were loaded on a $10 \%$ sodium dodecyl sulfate-polyacrylamide gel and subsequently transferred to a polyvinylidene difluoride membrane (Millipore, Billerica, MA, USA). Membranes were blocked in $5 \%$ non-fat dry milk in Tris-buffered saline (TBS) for $2 \mathrm{~h}$ at room temperature and then incubated with rabbit polyclonal anti-GRK2, anti-PUMA (Santa Cruz Biotechnology, Inc.), anti-caspase-3 (Epitomics, Burlingame, CA, USA) and anti-cleaved caspase-3 (Cell Signaling Technology, Inc., Danvers, MA, USA) antibodies overnight at $4^{\circ} \mathrm{C}$. Following three washes with TBS supplemented with Tween 20 (TBST), the membranes were incubated for $2 \mathrm{~h}$ with rabbit anti-human immunoglobulin $\mathrm{G}$ antibody (Cell Signaling Technology, Inc.) conjugated to horseradish peroxidase. Subsequent to washing with TBST, the membranes were exposed to enhanced chemiluminescent reagent (Millipore) for $1 \mathrm{~min}$ and then to Kodak X-ray film (Eastman Kodak, Rochester, NY, USA). Anti- $\beta$-actin antibody (Cell Signaling Technology, Inc.) was used to detect $\beta$-actin.
Statistical analysis. Data are presented as the mean \pm standard error. EFS was calculated using Kaplan-Meier analysis. The Student's t-test was performed for comparisons between two groups. Statistical analysis was conducted using SPSS 13.0 software (SPSS, Inc., Chicago, IL, USA). A value of $\mathrm{P}<0.05$ was considered to indicate a statistically significant difference.

\section{Results}

miR-125b is highly expressed in acute leukemia. To determine the differential expression pattern of miR-125b, qPCR was performed in bone marrow samples from patients with acute leukemia $(n=46)$, including those with AML and ALL. miR-125b expression was observed to be markedly higher in patients with acute leukemia compared with that in the healthy controls, demonstrating an average 4,375-fold increase (data not shown). Patients were divided into two risk groups, low and high, using median miR-125b expression. The median miR-125b expression value in the patients with acute leukemia was 85.24-fold higher than that in the samples from the healthy controls. Patients with an miR-125b expression value below the median were assigned to the low group and those with an expression value above the median were assigned to the high group. Among the 20 patients in the low group, two relapsed, two succumbed during therapy and nine did not achieve remission. A total of 13 patients had an event, and the remaining $35 \%$ showed an EFS. The average EFS was 6.2 months [95\% confidence interval (CI), 3.5-8.9]. Among the 26 patients in the high group, eight relapsed, three succumbed during therapy, one succumbed during bone marrow transplantation subsequent to achieving complete remission and 12 did not achieve remission. A total of 25 of the patients had an event, and the remaining 3.8\% showed an EFS. The average EFS was 3.3 months (95\% CI, 1.8-4.8). A significant difference was observed in the EFS between patients in the high group and those in the low group $(\mathrm{P}<0.05)$. However, the OS was not observed to be significantly different between the two groups $(\mathrm{P}=0.336)$ (Fig. 1).

miR-125b expression affects leukemia cell sensitivity to $D N R$. To investigate the association between miR-125b and DNR chemoresistance in leukemia cells, miR-125b was overexpressed in K562, THP-1 and Jurkat cells and knocked down in REH cells. K562, THP-1 and Jurkat cells were stably transduced with a murine stem cell virus (MSCV)-miR-125b vector to overexpress miR-125b in the cells (23). qPCR was used to assess the expression of miR-125b. In K562 cells, miR-125b expression was observed to be 3.2-fold higher in the cells transduced with MSCV-miR-125b compared with the cells transduced with an empty vector, and 4.9- and 4-fold higher in the THP-1 and Jurkat cells, respectively. The K562, THP-1 and Jurkat cells overexpressing miR-125b were treated with various doses of DNR. Overexpression of miR-125b was associated with a significant increase in the survival of K562, THP-1 and Jurkat cells (Fig. 2A-C). At DNR concentrations $\geq 0.1 \mu \mathrm{M}$, the survival of K562 and Jurkat cells was significantly higher in the miR-125b overexpression group than that in the control group $(\mathrm{P}<0.05)$. At DNR concentrations $\geq 0.01 \mu \mathrm{M}$, the survival of THP-1 cells was significantly higher in the miR-125b overexpression group than that in the control group $(\mathrm{P}<0.05)$. 
A

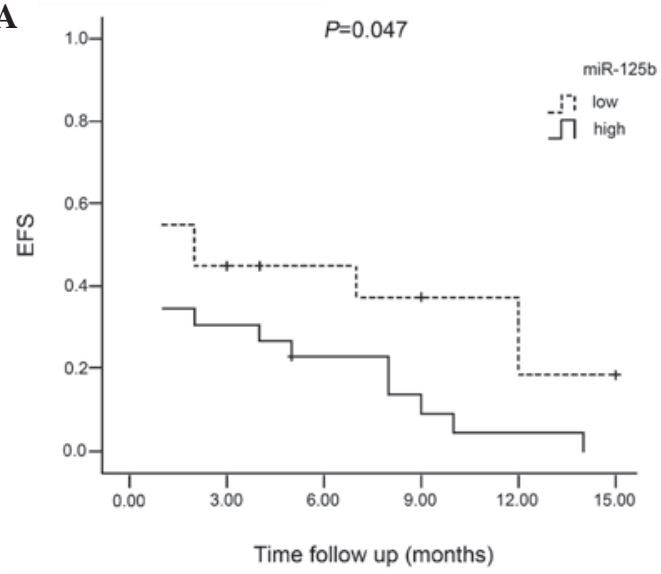

B

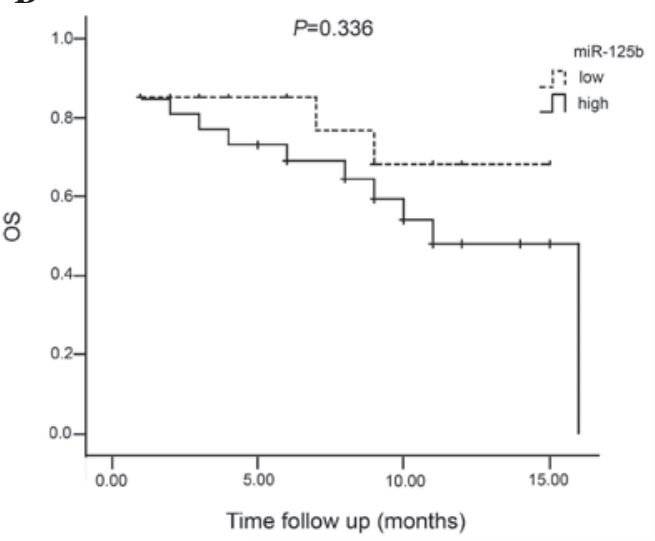

Figure 1. Correlation between miR-125b and prognosis in patients with acute leukemia. (A) EFS was significantly different between patients in the high miR-125b expression group and those in the low expression group $(\mathrm{P}<0.05)$. (B) OS was not significantly different between patients in the high miR-125b expression group and those in the low expression group $(\mathrm{P}=0.336)$. miR-125b; microRNA-125b; EFS, event-free survival; OS, overall survival.

A

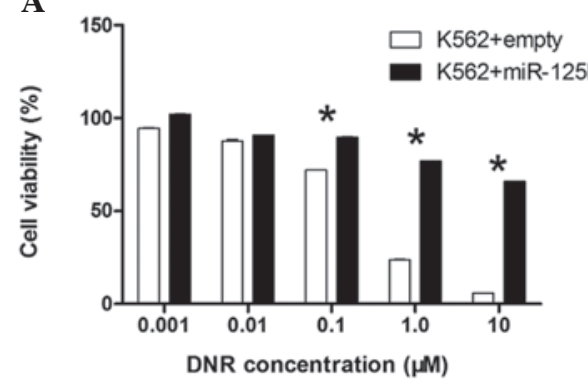

D

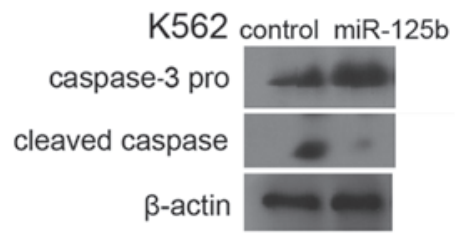

B

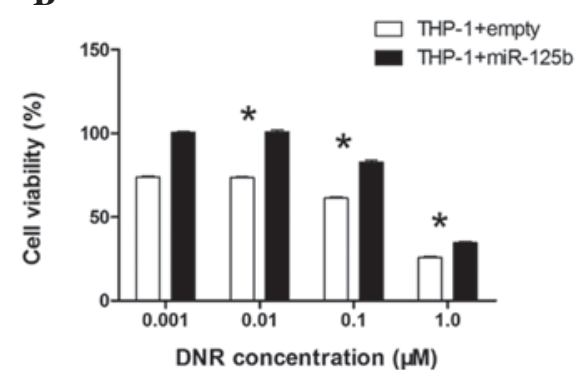

E

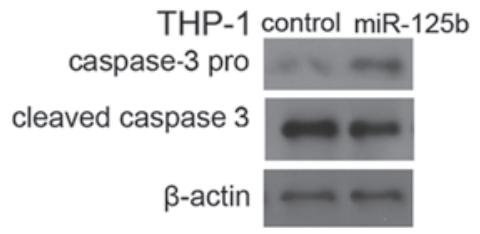

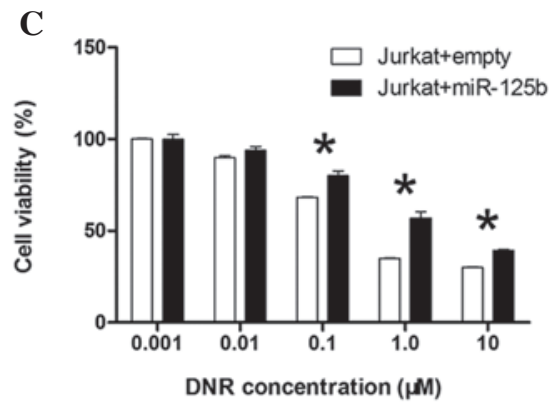

F

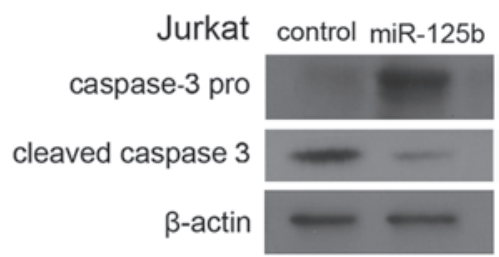

Figure 2. Overexpression of miR-125b sensitizes K562, THP-1 and Jurkat cells to DNR. (A) K562, (B) THP-1 and (C) Jurkat cells were transduced with MSCV-miR-125b or an empty vector and were treated with various doses of DNR. Cell viability was detected using luminescent cell viability assay. "P $<0.05$. (D-F) Representative western blot analyses showing caspase-3 pro and cleaved caspase-3 protein levels in (D) K562, (E) THP-1 and (F) Jurkat cells transduced with an empty vector or the miR-125b vector upon exposure to DNR. miR-125b, microRNA-125b; DNR, daunorubicin.

The effects of miR-125b-knockdown on REH cells were investigated using transduction with an miR-125b 'sponge' vector. The miR-125b 'sponge' vector effectively reduced the expression of miR-125b in REH cells, which was verified using qPCR. The expression of miR-125b in the miR-125b-knockdown REH cells was reduced by $75 \%$, compared with that in the control REH cells. The expression of miR-125b was significantly lower in the knockdown cells than that in the control cells $(\mathrm{P}<0.001)$ (Fig. 3A). Furthermore, knockdown of miR-125b was associated with a significantly decreased survival rate in the REH cells (Fig. 3B). At DNR concentrations $\geq 0.01 \mu \mathrm{M}$, miR-125b-knockdown REH cells exhibited a significantly lower survival rate than that of the control cells $(\mathrm{P}<0.05)$. This suggests that overexpression of miR-125b may contribute to DNR resistance in K562, THP-1 and Jurkat cells and that knockdown of miR-125b may contribute to DNR sensitivity in REH cells. Therefore, these findings suggest that a correlation exists between miR-125b expression and sensitivity to DNR in leukemia cells.

High expression of miR-125b inhibits apoptosis. In order to determine whether overexpression of miR-125b affects apoptosis, the release of activated caspase- 3 was assessed. Caspase- 3 pro and cleaved caspase- 3 protein levels were analyzed using western blot analysis. Upon treatment with DNR, K562, THP-1 and Jurkat cells overexpressing miR-125b exhibited higher expression of caspase-3 pro than the control group, and lower expression of cleaved caspase- 3 than the control group (Fig. 2D-F). Knockdown of miR-125b was observed to reduce the expression of caspase- 3 pro and increase that of cleaved caspase- 3 in REH cells compared with the expression in the control cells (Fig. 3C). These results show that overexpression 
A

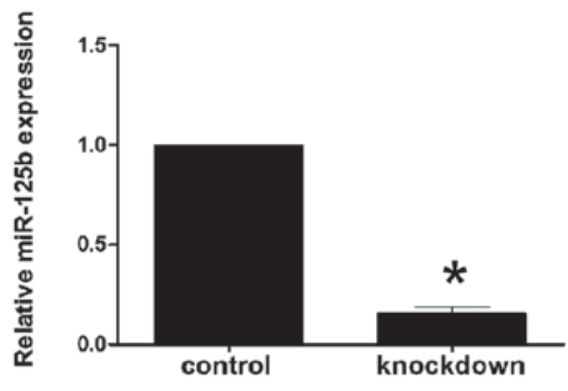

C

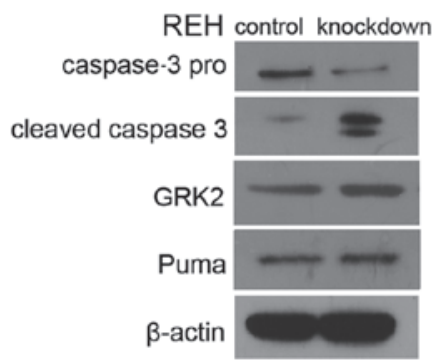

B

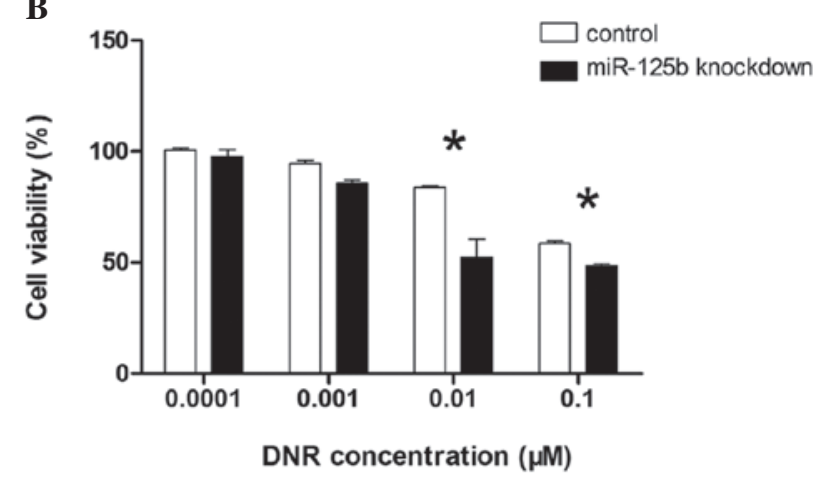

$\mathbf{E}$

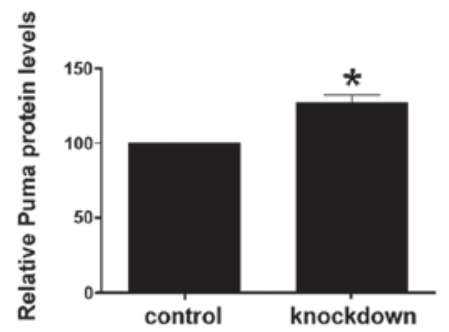

Figure 3. Knockdown of miR-125b in REH cells. (A) miR-125b expression was assessed using quantitative polymerase chain reaction analysis. ${ }^{*} \mathrm{P}<0.05$ vs. control. (B) REH and miR-125b-knockdown REH cells were transduced with miR-125b 'sponge' vector or an empty vector. The results are presented as the mean \pm standard error from three independent experiments. ${ }^{*}<0.05$. (C) Western blot analysis was performed using cell lysates and anti-GRK2 and -PUMA antibodies. $\beta$-actin was used as a loading control. Blots were representative of three independent experiments. (D and E) Relative expression of (D) GRK2 and (E) PUMA protein. "P<0.05 vs. control. miR-125b, microRNA-125b; GRK2, G protein-coupled receptor kinase 2; PUMA, p53-upregulated modulator of apoptosis.

of miR-125b induced DNR resistance through the inhibition of apoptosis and that the inhibition of miR-125b increased the sensitivity of REH cells to DNR by increasing apoptosis. These findings suggest that miR-125b increases the resistance of leukemia cells to DNR through inhibiting apoptosis.

miR-125b downregulates GRK2 and PUMA expression. To investigate the effect of miRNA on mRNA expression, miR-125b target mRNAs were obtained from the miRBase (http://www.mirbase.org) and the TargetScan (http://www. targetscan.org) databases (24). The correlation between miR-125b expression and the expression of its predicted target mRNAs, including the number of binding sites in the respective mRNAs and the variation in the correlation coefficients, were statistically analyzed. Among these potential gene targets, the present study focused on GRK 2 and PUMA, which may have effects on cell apoptosis (Fig. 3). It was hypothesized that GRK2 and PUMA are direct targets of miR-125b.

To investigate this hypothesis, HEK293T cells were separately cotransfected with miR-125b and either GRK2 or PUMA 3'UTR luciferase reporters. Transfections with control vector were performed in parallel. Cotransfection resulted in a 55 and $56.6 \%$ reduction in the reporter activity for GRK2 and PUMA, respectively $(\mathrm{P}<0.01$; Fig. 4A). These results demonstrate that GRK2 and PUMA 3'UTR are targets of miR-125b. The role of miR-125b in the regulation of GRK2 and PUMA expression in K562, THP-1 and Jurkat cells was then assessed. In the present study, it was hypothesized that overexpression of miR-125b was likely to reduce the GRK2 and PUMA protein expression. GRK2 and PUMA protein levels were observed to be lower in K562, THP-1 and Jurkat cells overexpressing miR-125b, compared with those in the corresponding control cells (Fig. 4). In the miR-125b-knockdown REH cells, GRK2 and PUMA protein levels were found to be significantly higher than those in the control cells (Fig. 3C-E). These findings show that GRK2 and PUMA are targets of miR-125b in K562, THP-1, Jurkat and REH cells.

GRK2 and PUMA have a key role in DNR resistance. GRK2 and PUMA siRNA was used to silence the expression of GRK2 and PUMA in K562 cells, respectively. GRK2 expression was observed to be significantly decreased upon transfection with GRK2 siRNA. Of note, K562 cells transfected with GRK2 siRNA exhibited a similar survival pattern to cells overexpressing miR-125b. The level of cleaved caspase-3 protein was also decreased upon DNR treatment in the cells transfected with GRK2 siRNA. Similarly, the expression of PUMA was significantly decreased following transfection of PUMA siRNA, and these cells exhibited a similar survival pattern to cells overexpressing miR-125b. The level of cleaved caspase- 3 protein was also observed to decrease upon DNR treatment in PUMA siRNA-transfected cells. These findings show that GRK2 and PUMA have a key role in DNR resistance (Fig. 5), and indicate that miR-125b contributes to DNR resistance through reducing the expression of GRK2 and PUMA in leukemia cells.

\section{Discussion}

To the best of our knowledge, this is the first study to show that miR-125b expression is significantly increased in patients 


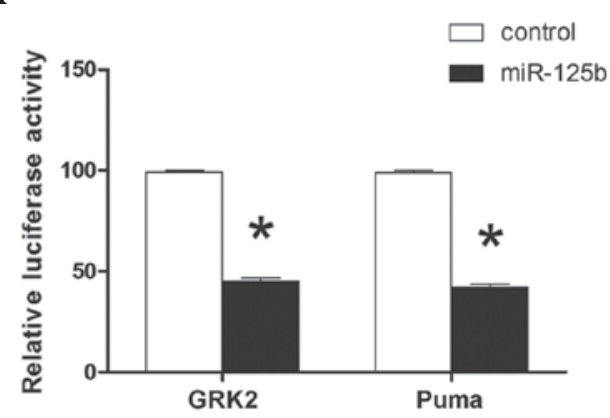

C

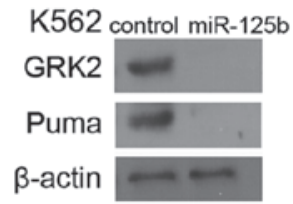

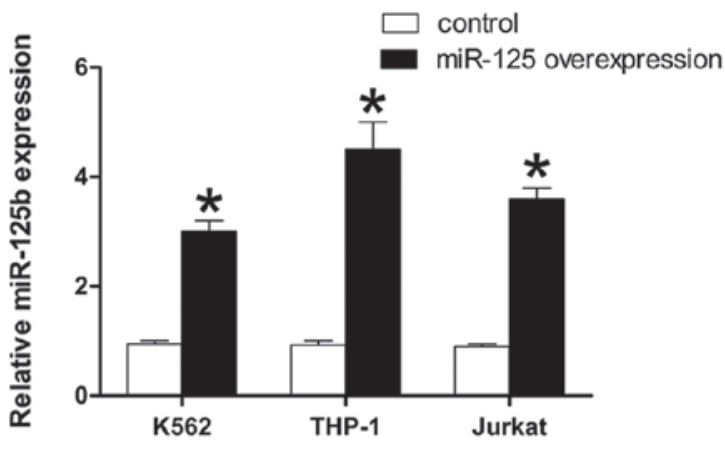

D

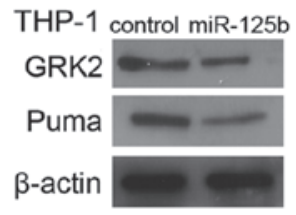

$\mathbf{E}$

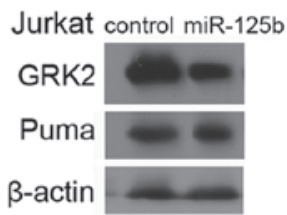

Figure 4. GRK2 and PUMA are direct targets of miR-125b. (A) The effect of miR-125b on GRK2 and PUMA expression was assessed using a luciferase reporter system. The miR-125b expression vector, together with the luciferase reporter vector or control vector, was cotransfected into the HEK293T cells. miR-125b bound to the GRK2 3' UTR and PUMA 3' UTR, resulting in reduced luciferase expression. Data are presented as the mean \pm standard error from three independent experiments. ${ }^{*} \mathrm{P}<0.05$ vs. control. (B) miR-125b expression was assessed using quantitative polymerase chain reaction analysis in control and miR-125b-overexpressing K562, THP-1 and Jurkat cells. "P<0.05 vs. control. (C-E) Western blot analysis was performed using cell lysates and anti-GRK2 and -PUMA antibodies. $\beta$-actin was used as a loading control. Relative GRK2 and PUMA protein expression is shown in (C) K562, (D) THP-1 and (E) Jurkat cells. Blots were representative of three independent experiments. miR-125b, microRNA-125b; GRK2, G protein-coupled receptor kinase 2; PUMA, p53-upregulated modulator of apoptosis; UTR, untranslated region.

A

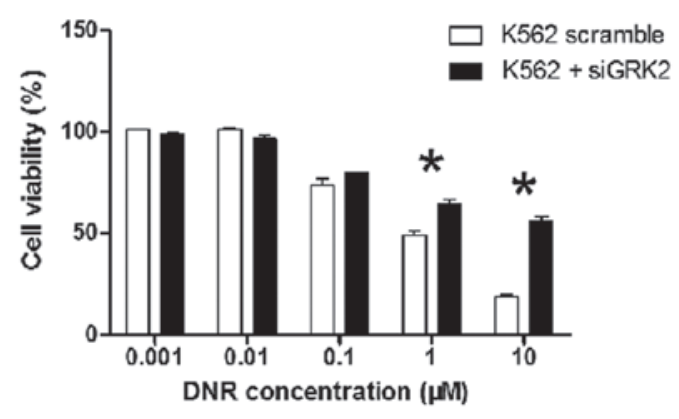

C

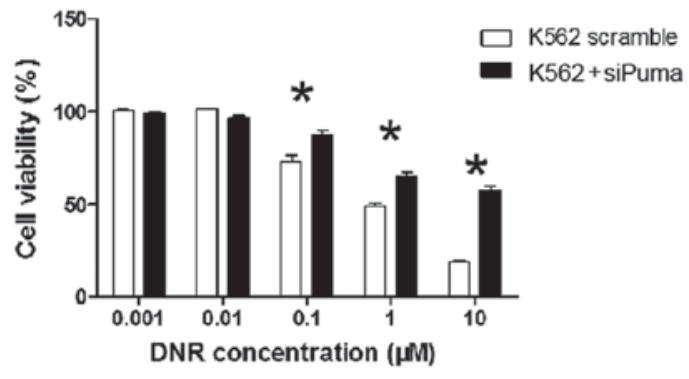

B

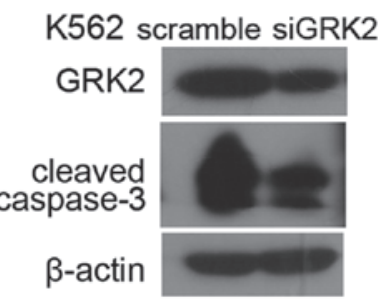

D

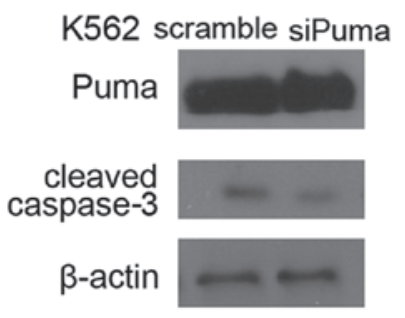

Figure 5. Silencing the expression of GRK2 and PUMA induces DNR resistance in leukemia cells. (A) GRK2 expression was silenced in K562 cells treated with various doses of DNR. Cell viability was detected using a luminescent cell viability assay. ${ }^{~} \mathrm{P}<0.05$. (B) Representative western blot analyses showing GRK2 and cleaved caspase-3 protein levels in GRK-2-silenced K562 cells upon exposure to DNR. (C) PUMA expression was silenced in K562 cells treated with various doses of DNR. Cell viability was detected using a luminescent cell viability assay. "P $<0.05$. (D) Representative western blot analyses showing PUMA and cleaved caspase-3 protein levels in PUMA-silenced K562 cells upon expsosure to DNR. GRK2, G protein-coupled receptor kinase 2; PUMA, p53-upregulated modulator of apoptosis; DNR, daunorubicin; siRNA, small interfering RNA.

who undergo an event $(\mathrm{P}<0.05)$ in acute leukemia, with the exception of those classified as M3 (promyelocytic leukemia). Patients in remission may have a longer EFS than patients who undergo an event. DNR-induced drug resistance is associated with the upregulation of miR-125b in K562, THP-1 and Jurkat cell lines. Furthermore, miR-125b may regulate the survival 
of leukemia cells by targeting GRK2 and PUMA proteins and inducing changes in apoptosis in K562, THP-1, Jurkat and REH cells.

Overexpression of miR-125b occurs in patients with myelodysplasia, megakaryoblastic leukemia (15) and APL (18), as well as those with AML who carry the $t(2 ; 11)$ (p21;q23) translocation (13). High expression of miR-125b is correlated with treatment response, as well as relapse in pediatric APL (18). miR-125b may predict poor prognosis in non-small-cell lung (10) and colorectal cancer (12). The present study employed primary patients and relapse or no-remission patients; therefore, the average expression level of miR-125b was extremely high. Based on induction therapy, the improved survival time of patients with APL and the short follow-up duration in the present study, patients with APL were not included in this investigation. In the present study, event-free patients were observed to exhibit longer EFS than therapy-failure patients. Although there was no significant difference in OS between patients in remission and event patients, patients in remission were found to have an increased survival compared with other patients. These findings suggest that miR-125b may be an important prognostic marker for patients with acute leukemia.

The association between miR-125b and drug resistance has been investigated in numerous types of human cancer. However, contradictory data exist regarding the expression of miR-125b in different tumor types, and miR-125b has been reported to have different roles in drug sensitivity and drug resistance. miR-125b is downregulated in resistant Ehrlich ascites tumor cells (25) and has been shown to increase the apoptotic response to cisplatin treatment in breast cells (26). Conversely, miR-125b has been found to be upregulated in Taxol-resistant cancer (9), doxorubicin-resistant Ewing sarcoma (27), cisplatin-resistant ovarian cancer (28) and AML (18) cells. In the present study, miR-125b upregulation was found to increase cell survival compared with that in the control cells, whereas miR-125b downregulation was observed to decrease cell survival. These findings indicate that high expression of miR-125b induces resistance to DNR treatment.

The mechanism underlying patient resistance to chemotherapeutic treatment is yet to be elucidated. Therefore, identifying the cause of drug resistance and developing biomarkers are critical. Anthracyclines have been shown to intercalate with DNA and indirectly inhibit the activity of the enzyme topoisomerase II, resulting in DNA strand breaks and induction of apoptosis (29). In the present study, it was hypothesized that miR-125b induced DNR resistance in leukemia cells through regulating apoptosis. Previous studies have shown that members of the B-cell lymphoma (Bcl)-2 family and other factors involved in apoptosis are important targets of miR-125. Numerous proteins have been shown to affect apoptosis, including anti-apoptotic members of the Bcl-2 family, such as Bcl-w (30), Bcl-2 (31), myeloid cell leukemia sequence $1(30,32)$ and Bcl-2-antagonist/killer (Bak)-1 (9,28,30,32), acting as the Bcl-2 homologous antagonist, and pro-apoptotic targets, including P53 (33), tumor protein p53-inducible nuclear protein 1 (TP53INP1) (34), tumor necrosis factor $\alpha$-induced protein 3 (35) and p38 $\alpha$ (36). High miR-125 expression has been shown to downregulate Bak-1 and TP53INP1, and consequently protects cells from apoptosis and promotes tumorigenesis (37). In the present study, GRK2 and PUMA were identified to be direct targets of miR-125b in leukemia cells. GRK2 and PUMA protein expression was found to be downregulated by miR-125b in K562, THP-1 and Jurkat cells. Furthermore, miR-125b upregulation inhibited DNR-induced apoptosis in K562, THP-1 and Jurkat cells. GRK2 and PUMA protein expression was also upregulated by $\mathrm{miR}-125 \mathrm{~b}$ in REH cells. Downregulation of miR-125b increased cell apoptosis following treatment with DNR. These results indicate that miR-125b downregulated GRK2 and PUMA, which inhibited apoptosis and induced leukemia cell resistance to DNR. However, the detailed mechanism underlying this action is yet to be elucidated.

In conclusion, this study has shown that DNR resistance is associated with miR-125b upregulation and the consequent downregulation of the miR-125b target genes GRK2 and PUMA. Therefore, upregulation of miR-125b may inactivate the caspase pathway and inhibit apoptosis, and dysregulation of miR-125b may lead to the acquisition of DNR resistance in AML. Of note, miR-125b expression was found to be associated with disease development and treatment response. These results suggest that miR-125b may represent a potential biomarker for predicting the effect of chemotherapy in patients with leukemia.

\section{Acknowledgements}

This study was supported by a grant from the Shanghai Jiaotong University Affiliated Shanghai First People's Hospital (no. 061138). The authors would like to thank the staff of the Department of Hematology of Shanghai Jiaotong University Affiliated First People's Hospital.

\section{References}

1. Kimby E, Nygren P and Glimelius B; SBU-group. Swedish Council of Technology Assessment in Health Care: A systematic overview of chemotherapy effects in acute myeloid leukaemia. Acta Oncol 40: 231-252, 2001.

2. Tallman MS, Gilliland DG and Rowe JM: Drug therapy for acute myeloid leukemia. Blood 106: 1154-1163, 2005.

3. Pulte D, Gondos A and Brenner H: Expected long-term survival of patients diagnosed with acute myeloblastic leukemia during 2006-2010. Ann Oncol 21: 335-341, 2010.

4. Bartel DP: MicroRNAs: genomics, biogenesis, mechanism, and function. Cell 116: 281-297, 2004.

5. Ambros V: The functions of animal microRNAs. Nature 431: 350-355, 2004.

6. Lim LP, Lau NC, Garrett-Engele P, et al: Microarray analysis shows that some microRNAs downregulate large numbers of target mRNAs. Nature 433: 769-773, 2005.

7. Lu J, Getz G, Miska EA, et al: MicroRNA expression profiles classify human cancers. Nature 435: 834-838, 2005.

8. Kim JK, Noh JH, Jung KH, et al: Sirtuin7 oncogenic potential in human hepatocellular carcinoma and its regulation by the tumor suppressors MiR-125a-5p and MiR-125b. Hepatology 57: 1055-1067, 2013.

9. Zhou M, Liu Z, Zhao Y, et al: MicroRNA-125b confers the resistance of breast cancer cells to paclitaxel through suppression of pro-apoptotic Bcl-2 antagonist killer 1 (Bak1) expression. J Biol Chem 285: 21496-21507, 2010.

10. Yuxia M, Zhennan T and Wei Z: Circulating miR-125b is a novel biomarker for screening non-small-cell lung cancer and predicts poor prognosis. J Cancer Res Clin Oncol 138: 2045-2050, 2012.

11. Kappelmann M, Kuphal S, Meister G, Vardimon L and Bosserhoff AK: MicroRNA miR-125b controls melanoma progression by direct regulation of c-Jun protein expression. Oncogene 32: 2984-2991, 2013. 
12. Nishida N, Yokobori T, Mimori K, et al: MicroRNA miR-125b is a prognostic marker in human colorectal cancer. Int J Oncol 38: 1437-1443, 2011.

13. Bousquet M, Quelen C, Rosati R, et al: Myeloid cell differentiation arrest by miR-125b-1 in myelodysplastic syndrome and acute myeloid leukemia with the $\mathrm{t}(2 ; 11)(\mathrm{p} 21 ; \mathrm{q} 23)$ translocation. J Exp Med 205: 2499-2506, 2008.

14. Enomoto Y, Kitaura J, Hatakeyama K, et al: E $\mu / \mathrm{miR}-125 b$ transgenic mice develop lethal B-cell malignancies. Leukemia 25: 1849-1856, 2011.

15. Klusmann JH, Li Z, Böhmer K, et al: miR-125b-2 is a potential oncomiR on human chromosome 21 in megakaryoblastic leukemia. Genes Dev 24: 478-490, 2010.

16. Gefen N, Binder V, Zaliova M, et al: Hsa-mir-125b-2 is highly expressed in childhood ETV6/RUNX1 (TEL/AML1) leukemias and confers survival advantage to growth inhibitory signals independent of p53. Leukemia 24: 89-96, 2010.

17. Calin GA, Liu CG, Sevignani C, et al: MicroRNA profiling reveals distinct signatures in B cell chronic lymphocytic leukemias. Proc Natl Acad Sci USA 101: 11755-11760, 2004

18. Zhang $\mathrm{H}$, Luo XQ, Feng DD, et al: Upregulation of microRNA-125b contributes to leukemogenesis and increases drug resistance in pediatric acute promyelocytic leukemia. Mol Cancer 10: 108, 2011

19. Schotte D, De Menezes RX, Akbari Moqadam F, et al: MicroRNA characterize genetic diversity and drug resistance in pediatric acute lymphoblastic leukemia. Haematologica 96 703-711, 2011

20. Döhner H, Estey EH, Amadori S, et al; European LeukemiaNet: Diagnosis and management of acute myeloid leukemia in adults: recommendations from an international expert panel, on behalf of the European LeukemiaNet. Blood 115: 453-474, 2010.

21. Livak KJ and Schmittgen TD: Analysis of relative gene expression data using real-time quantitative PCR and the 2(-Delta Delta C(T)) method. Methods 25: 402-408, 2001.

22. Starczynowski DT, Kuchenbauer F, Argiropoulos B, et al: Identification of miR-145 and miR-146a as mediators of the 5q-syndrome phenotype. Nat Med 16: 49-58, 2010.

23. Hughes MS, Yu YY, Dudley ME, et al: Transfer of a TCR gene derived from a patient with a marked antitumor response conveys highly active T-cell effector functions. Hum Gene Ther 16 : 457-472, 2005.

24. Stark A, Brennecke J, Russell RB and Cohen SM: Identification of Drosophila MicroRNA targets. PLoS Biol 1: 397-409, 2003.
25. Husted S, Søkilde R, Rask L, et al: MicroRNA expression profiles associated with development of drug resistance in Ehrlich ascites tumor cells. Mol Pharm 8: 2055-2062, 2011.

26. Rajabi H, Jin C, Ahmad R, McClary C, Joshi MD and Kufe D Mucin 1 oncoprotein expression is suppressed by the miR-125b oncomir. Genes Cancer 1: 62-68, 2010.

27. Iida K, Fukushi J, Matsumoto Y, et al: miR-125b develops chemoresistance in Ewing sarcoma/primitive neuroectodermal tumor. Cancer Cell Int 13: 21, 2013.

28. Kong F, Sun C, Wang Z, et al: miR-125b confers resistance of ovarian cancer cells to cisplatin by targeting pro-apoptotic Bcl-2 antagonist killer 1. J Huazhong Univ Sci Technolog Med Sci 31: 543-549, 2011.

29. Richardson DS and Johnson SA: Anthracyclines in haematology: preclinical studies, toxicity and delivery systems. Blood Rev 11 201-223, 1997.

30. Gong J, Zhang JP, Li B, et al: MicroRNA-125b promotes apoptosis by regulating the expression of Mcl-1, Bcl-w and IL-6R. Oncogene 32: 3071-3079, 2013.

31. Shi L, Zhang S, Feng K, et al: MicroRNA-125b-2 confers human glioblastoma stem cells resistance to temozolomide through the mitochondrial pathway of apoptosis. Int J Oncol 40: 119-129, 2012.

32. Balakrishnan A, Stearns AT, Park PJ, et al: Upregulation of proapoptotic microRNA mir-125a after massive small bowel resection in rats. Ann Surg 255: 747-753, 2012.

33. Zeng CW, Zhang XJ, Lin KY, et al: Camptothecin induces apoptosis in cancer cells via microRNA-125b-mediated mitochondrial pathways. Mol Pharmacol 81: 578-586, 2012.

34. Jiang F, Liu T, He Y, et al: MiR-125b promotes proliferation and migration of type II endometrial carcinoma cells through targeting TP53INP1 tumor suppressor in vitro and in vivo. BMC Cancer 11: 425, 2011.

35. Kim SW, Ramasamy K, Bouamar H, Lin AP, Jiang D and Aguiar RC: MicroRNAs miR-125a and miR-125b constitutively activate the NF- $\kappa \mathrm{B}$ pathway by targeting the tumor necrosis factor alpha-induced protein 3 (TNFAIP3, A20). Proc Natl Acad Sci USA 109: 7865-7870, 2012.

36. Tan G, Niu J, Shi Y, Ouyang H and Wu ZH: NF-kB-dependent microRNA-125b up-regulation promotes cell survival by targeting p38 $\alpha$ upon ultraviolet radiation. J Biol Chem 287: 33036-33047, 2012.

37. Bousquet M, Nguyen D, Chen C, et al: MicroRNA-125b transforms myeloid cell lines by repressing multiple mRNA. Haematologica 97: 1713-1721, 2012. 